\title{
PAPER
}

\section{The influences and outcomes of phonological awareness: a study of MA, PA and auditory processing in pre-readers with a family risk of dyslexia}

\section{Jeremy M Law, ${ }^{1}$ Jan Wouters ${ }^{2}$ and Pol Ghesquière ${ }^{1}$}

1. Parenting and Special Education Research Unit, KU Leuven, Belgium

2. Laboratory for Experimental ORL, KU Leuven, Belgium

\begin{abstract}
The direct influence of phonological awareness $(P A)$ on reading outcomes has been widely demonstrated, yet $P A$ may also exert indirect influence on reading outcomes through other cognitive variables such as morphological awareness (MA). However, PA's own development is dependent and influenced by many extraneous variables such as auditory processing, which could ultimately impact reading outcomes. In a group of pre-reading children with a family risk of dyslexia and low-risk controls, this study sets out to answer questions surrounding PA's relationship at various grain sizes (syllable, onset/rime and phoneme) with measures of auditory processing (frequency modulation ( $F M)$ and an amplitude rise-time task $(R T)$ ) and $M A$, independent of reading experience. Group analysis revealed significant differences between high-and low-risk children on measures of MA, and PA at all grain sizes, while a trend for lower RT thresholds of high-risk children was found compared with controls. Correlational analysis demonstrated that MA is related to the composite PA score and syllable awareness. Group differences on MA and PA were re-examined including $P A$ and $M A$, respectively, as control variables. Results exposed $P A$ as a relevant component of MA, independent of reading experience.
\end{abstract}

\section{Research highlights}

- This is the first paper to report together morphological awareness (MA), phonological awareness (PA) and auditory processing in a pre-reading sample of high-risk children.

- Demonstrates an MA deficit in children with a family risk of dyslexia prior to reading instruction.

- Findings of a significant difference between controls and children with a family risk of dyslexia in auditory processing skills related to rise-time awareness.

- Early pre-reading MA is shown to be a function of PA.

\section{Introduction}

Developmental dyslexia, a hereditary neurodevelopmental disorder, is characterized by severe reading and/or spelling impairments that are persistent throughout life (Vellutino, Fletcher, Snowling \& Scanlon, 2004). Research has suggested that the poor decoding abilities observed in people with dyslexia stem from a cognitive deficit in the development of, and/or access to, phoneme representations (Snowling, 2000; Tønnessen, 1997; but see Ramus \& Szenkovits, 2008). Such observations have led to the development of the phonological deficit theory of dyslexia. Evidence of the past decade has suggested that a more fundamental deficit in auditory processing is underlying the phonological deficit, leading to the auditory temporal processing deficit theory of dyslexia (Boets, Wouters, Van Wieringen \& Ghesquière, 2007; Goswami, Thomson, Richardson, Stainthorp, Hughes et al., 2002; Tallal, 1980). This theory postulates that a deficit in the processing of dynamic auditory stimuli negatively impacts the perception of syllables and phonemes, leading to a disruption of the development 
of suitable phonological representations (Poelmans, Luts, Vandermosten, Boets, Ghesquière et al., 2011).

Although phonological processing deficits have been found to describe a significant portion of the variance in reading by dyslexic people, a large proportion of the variance still remains unexplained. As a result recent theories of dyslexia have highlighted the possible existence of a multitude of interacting deficits. It is thought that individuals' expressed behavioural deficits may be a function of multiple cognitive factors acting as risk or protective factors, independently or in conjunction with the phonological deficit (Pennington, 2006). Such insights have led the exploration of alternative cognitive variables to account for the observed problems in the reading and spelling of persons with dyslexia. Morphological awareness (MA) is one such variable that has begun to be recognized as a contributing variable in word recognition, independent of orthographic processing, phonological awareness, rapid automatized naming, and vocabulary (Carlisle, 2000; Deacon \& Kirby, 2004; Kirby, Deacon, Bowers, Izenberg, Wade-Woolley et al., 2012). Yet disagreement exists as to the role of MA within the dyslexic population. Is MA merely a function of the dyslexic readers' poor PA skills and reduced reading experience (Carlisle, Colé \& Sopo, 2004; Deacon, Parrila \& Kirby, 2008), or does MA play a possible compensatory role in the poor reading abilities of persons with dyslexia (Elbro \& Arnbak, 1996; Law, Wouters \& Ghesquière 2015; Leikin \& Zur Hagit, 2006)? A better understanding of how early MA relates to the phonological skills of children with dyslexia during their pre-reading phase could help in disentangling the relationship of reading experience, PA and MA.

The aim of this study is twofold. First, in a group of pre-reading children with a family risk of dyslexia and low-risk controls, we will analyse how the phonological deficit is expressed at various grain sizes of PA (onset/ rime, syllables and phonemes) and how PA proficiency at each of these grain sizes relates to measures of auditory temporal processing. Such analysis allows for an assessment of the auditory temporal processing theory of dyslexia and helps to demonstrate the relationship between measures of auditory temporal processing and PA proposed by the theory, thus answering questions about the role of deviant auditory processing as a risk factor for a deficit in phonological development.

Secondly, the relationship between the various PA and MA skills will be explored in an attempt to shed light on the pre-reading relationship of MA and PA, specifically addressing questions regarding the role of early PA on the development of MA.

\section{Phonological awareness}

A child's phonological awareness (PA), the ability to recognize, isolate and manipulate the basic sound units of a language, begins to develop early in life. Prior to reading instruction, the development of phonological awareness begins with the explicit awareness of larger grain size units (syllables and onset/rimes). Only after explicit instruction do children develop smaller grain size representations at the level of the phoneme, thus establishing phonemic awareness (Ziegler \& Goswami, 2005). This developmental progression has been shown to have neurological foundations (Vanvooren, Poelmans, Hofmann, Ghesquière \& Wouters, 2014). In a 2014 study of pre-reading children, Vanvooren and colleagues noted mature hemispheric specialization for processing syllable rate modulations in pre-reading children, while hemispheric specialization for phoneme rate modulation processing appeared to be still developing.

Phonological awareness skills have been shown to be vital in later reading and spelling achievement across both transparent and opaque orthographies. Pre-reading phonological awareness has been demonstrated to account for $40-60 \%$ of the later reading achievement of kindergarten children (Bryant, MacLean, Bradley \& Crossland, 1990; Caravolas, Hulme \& Snowling, 2001; Wagner, Torgesen \& Rashotte, 1994).

Deficits in phonological awareness are often characteristic of dyslexia. Early pre-reading deficits in PA have been observed in children at high risk of, or later diagnosed with, dyslexia (Elbro, Borstrøm \& Petersen, 1998; Pennington \& Lefly, 2001; Snowling, Gallagher \& Frith, 2003). This deficit has been shown to persist after the onset of reading instruction (Boets, Vandermosten, Poelmans, Luts, Wouters et al., 2011; Dandache, Wouters \& Ghesquière, 2014; Snowling, Muter \& Carroll, 2007). Research over the past decade has begun to suggest that the observed phonological deficit of dyslexic readers is linked to a more fundamental deficit in auditory processing (Boets et al., 2007).

\section{Auditory temporal processing and dyslexia}

Spoken-language comprehension and processing is dependent on the accurate isolation and interpretation of meaningful units of speech such as words, sentences or utterances. Such high-level perceptual units are an amalgamation of basal acoustic-phonetic cues that can be categorized within various time scales corresponding to various phonological grain size units. For example, syllable recognition is reliant on time windows corresponding to a range of $3-7 \mathrm{~Hz}$ while shortened time scales of $12-50 \mathrm{~Hz}$ corresponds to segmental 
information related with phoneme identity (Goswami, Fosker, Huss, Mead \& Szucs, 2011; Obrig, Rossi, Telkemeyer \& Wartenburger, 2010). It is believed that during the pre-literate phase of development, the attainment of well-specified sub-lexical phonological representations is dependent upon the accurate perception and processing of acoustic cues signalling syllable and/or phoneme specific time windows in speech. Therefore, it is reasonable to assume that an individual with an impairment relating to the processing of these low-level auditory temporal cues would be limited in their ability to reflect upon - and isolate - the basal phonological information of words in speech, thus resulting in inaccurate phonological representations (Boets et al., 2007; Goswami et al., 2002; Tallal, 1980).

In the past decade, various auditory parameters have been used in research to explore the relationship between impaired auditory processing and the phonological deficit associated with dyslexia. In a recent review, Hämäläinen and colleagues showed that group differences between dyslexic people and controls were most often observed in studies that utilized slow rate $(<60 \mathrm{~Hz})$ sound parameters of frequency modulation $(\mathrm{FM})$ and measures of amplitude rise time (RT) (Hämäläinen, Salminen \& Leppänen, 2013). In this review, encompassing an age range of 8.8-37 years, significant group differences between dyslexics and controls were found for $100 \%$ of the studies utilizing RT stimuli and $92 \%$ of the FM studies.

Significant group differences have been shown to exist between dyslexics and controls on measures of FM detection. Such studies have revealed that dyslexics have a reduced sensitivity compared to the control group, thus demonstrating the FM task's ability to differentiate between adult, school-aged children and pre-reading dyslexics from normal readers (Boets et al., 2007; Ramus, Rosen, Dakin, Day, Castellote et al., 2003; Witton, Stein, Stoodley, Rosner \& Talcott, 2002; Witton, Talcott, Hansen, Richardson, Griffiths et al., 1998). In addition, phonological decoding skills of adults with dyslexia and controls have been found to significantly correlate with FM sensitivity of 2 and $40 \mathrm{~Hz}$ (Witton et al., 1998). Similarly, Hämäläinen et al. (2013) noted eight separate studies that reported correlations between FM detection thresholds and reading and/or spelling skills, while three studies were unable to replicate these results (Dawes \& Bishop, 2009; Heath, Bishop, Hogben \& Roach, 2006; Van Ingelghem, Boets, van Wieringen, Onghena, Ghesquière et al., 2005).

Unlike FM tasks, amplitude rise time (RT) tasks assess an individual's perceptual sensitivity to the rate of change of the onset of the amplitude envelope, a temporal cue associated with the signalling of the onset of new syllables. Detection of such linguistic markers is thought to be important in speech perception and in early phonological development (e.g. Goswami et al., 2011; Goswami et al., 2002; Poelmans et al., 2011). Detection of such cues has been shown to be significantly associated with the reading, writing and phonological skills of adults (Corriveau, Pasquini \& Goswami, 2007; Hämäläinen, Leppänen, Torppa, Müller \& Lyytinen, 2005; Law, Vandermosten, Ghesquière \& Wouters, 2014) and children (Corriveau, Goswami \& Thomson, 2010; Goswami et al., 2002; Poelmans et al., 2011). One of the few studies that has assessed RT in a group of preschool children demonstrated the early importance of RT sensitivity in developing phonological awareness skills, specifically rhyme awareness (Corriveau et al., 2010).

\section{Morphological awareness}

Morphological awareness (MA), often described as the explicit awareness and ability to manipulate and reflect upon the morphemic structure of words, has been shown to exist already in pre-reading children (Berko, 1958; Carlisle, 1995; Lyytinen \& Lyytinen, 2004). Recent studies have recognized MA as a contributing variable in word recognition and reading comprehension, independent of orthographic processing, phonological awareness, RAN, and vocabulary (Carlisle, 2004; Deacon \& Kirby, 2004; Kirby et al., 2012; Roman, Kirby, Parrila, Wade-Woolley \& Deacon, 2009).

In English, morphological awareness aids in the identification, comprehension and pronunciation of words by the analysis of their component morphemes. A review paper by Bowers, Kirby and Deacon (2010) highlighted how the implicit awareness of morphemic boundaries influences the pronunciation of letter sequences, thus aiding decoding. Bowers et al. presented the boundary between 'ea' as an example. In the case of the word 'reach', which comprises a single morpheme, the letter combination is segmented and processed as one phoneme. While for 'react' the 'ea' is pronounced separately due to its placement in two adjacent morphemes. In addition, comprehension is aided through syntactic and lexical information provided by the suffix and prefix, allowing the reader to learn new morphologically complex words (Nagy, Berninger \& Abbott, 2006).

Research has shown that MA begins developing prior to reading instruction. Studies utilizing oral tasks have demonstrated morphological awareness in pre-reading children of 4 years old (Berko, 1958). Berko used an oral, non-word completion task that required children to apply morphemes on a target non-word to complete the 
sentence (i.e. Here is one WUG; now look there are two of them, there are two [WUGS]). Children as young as 4 years old were able to complete this task, yet some struggled on tasks requiring more complex morphological transformations, indicating an incomplete development of their morphological awareness. Often, anecdotal evidence is cited as evidence of preschool children possessing an implicit ability to produce some derived forms, such as 'flyable' or 'gooder'.

Carlisle and Fleming (2003) showed that morphological awareness progressively develops with age and through increased print exposure. Reading experience introduces a wider range of morphologically complex words, which stimulates and expands an individual's morphological awareness (Nagy \& Anderson, 1984). Five- to 6-year-old children were found to be able to decompose familiar words into their constituent morphemes but could not explicitly account for the lexical or syntactic information contained within the affixes. However, by grade 3, these skills had improved (Carlisle $\&$ Fleming, 2003). Studies have shown that the contribution of morphological awareness to reading ability also changes and strengthens with development (i.e. Singson, Mahony \& Mann, 2000).

Cunningham and Carroll (2013) highlighted two distinct theories that have been put forward in the literature regarding the early development and predictors of MA. The first depicts early MA development as a function of a broad base of oral language skills. As a child's oral vocabulary grows, they are able to link generalizable morphological units that are shared between words. The isolation and extraction of these units, therefore, allow for the development of an awareness of morphemes (i.e. -ing as in 'jumping' and 'running'). Correlational evidence has been provided supporting this theory (Kieffer \& Lesaux, 2012; Nagy et al., 2006). However, directionality cannot be implied through correlations. In a study of preschool children, McBride-Chang, Wagner, Muse, Chow and Shu (2005) provided evidence of a reciprocal relationship between vocabulary and morphology, showing that gains in one variable led to gains in the other.

An alternative theory, discussed by Carlisle and Nomanbhoy (1993) and later elaborated by Chiat (2001), implicates a child's phonological skills in MA development. The theory argues that phonological processing is not merely limited to the lexical and sub-lexical segmentation and representation, yet it is implicated in the development of syntactic and semantic aspects of oral language, thus influencing MA development. Chiat reasoned that while acquiring language, young children are presented with a stream of speech set in, and related to, the context of a specific environment. The child is required to segment the stream of speech recurrently until (s)he is able to identify usable meaningful phonological components. In addition, the child must understand the context and environment in which the phonological unit is isolated from speech. This awareness permits the creation of mappings between potential semantic, syntactic and phonological information, which allows for morphological learning to take place. For example, in the acquisition of the morpheme -ing, the child must first be able to recognize and process the phonological information of this speech unit when spoken. Also, the child must recognize that this speech unit often co-occurs with action being undertaken presently in the environment (i.e. 'look at the man jumping' while a man is seen jumping). As children's phonological awareness develops and they become aware of the phonemic structure of language, further morphological learning is permitted. For instance, greater phonological sophistication aids in the learning of morphophonemic rules that are required to understand the phonological shifts often made during the application of a suffix (e.g. divide and division, invade and invasion; Carlisle \& Nomanbhoy, 1993).

This theory has been supported by Cunningham and Carroll (2013) who found that pre-reading phonological processing predicted grade one morphological awareness and strategy use for spelling and non-word reading. Similarly, Carlisle and Nomanbhoy (1993) found that kindergarten PA (as measured through syllable and phoneme deletion) could predict expressive MA after controlling for vocabulary. Intervention studies of typically developing kindergarten children and those with speech impairments have also demonstrated gains in MA skills through PA instruction (Casalis \& Colé, 2009; Kirk \& Gillon, 2007).

Based on these competing theories, it is reasonable to assume that the phonological impairment of children with dyslexia would result in poor morphological development and early MA attainment, or in contrast, MA could independently develop in the context of literacy acquisition and the semantic and syntactic units conveyed in oral language (Casalis, Colé \& Sopo, 2004).

People with dyslexia have been found to have significantly poorer performance on many morphological production tasks when compared with chronologically age-matched controls (Casalis et al., 2004; Fowler \& Liberman, 1995; Shankweiler, Crain, Katz, Fowler, Liberman et al., 1995). Yet, when compared with reading-age matched controls, no differences could be found (Casalis et al., 2004; Elbro, 1989; Fowler \& Liberman, 1995). The observed phonological impairments of dyslexic children have been shown to prevent the explicit segmentation of affixes while leaving productive 
morphological knowledge development unaffected, suggesting a dependency of morphological awareness development on reading experience and phonological skills (Casalis et al., 2004). Because the PA deficit of people with dyslexia greatly influences later reading experience, the study of a dyslexic person's morphological awareness in a pre-reading population would be required to determine the relationship the phonological deficit has with the development of MA in dyslexic children.

\section{Objectives and goals of the study}

The objective of this study is to explore pre-reading MA, PA and measures of auditory temporal processing in children with a family risk of dyslexia. First, we will explore how our high- and low-risk groups fit with the current cognitive theories of dyslexia, as pertaining to poor phonological representations. Group differences of the three aspects of phonological processing, phonological awareness (PA), verbal short-term memory, and lexical retrieval (Wagner \& Torgesen, 1987) will be assessed to explore whether high-risk groups demonstrate a deficit in the phonological domain, as predicted by the phonological deficit theory of dyslexia.

In addition, the study will assess whether the phonological deficit is present at various grain sizes of PA in a pre-reading population. Further, the relationship between different measures of auditory temporal processing (RT and FM) and the various grain sizes of PA will be studied in order to test the proposed auditory temporal processing deficit theory of dyslexia. This study will also evaluate pre-reading MA in children of high-risk and low-risk groups and its relationship to PA at various grain sizes. Older, literate dyslexic children are found to differ from age-matched controls on MA measures, yet no differences in their MA performance are found when compared with reading age matched controls. Poor MA skills observed in populations of dyslexic people are often attributed to their reading experience, and/or as a result of their poor PA. By examining the relationship of PA and MA prior to formal reading instruction, we can help disentangling MA's debated relationship with reading experience and PA.

\section{Method}

\section{Participants}

A group of 58 preschool children ranging in age from 4 to 5 years old and attending Senior Kindergarten (SK) in the Ontario, Canada public school system were selected for the study. Children were classified as either being at high- risk (HR) for developing dyslexia $(N=23)$, or as low risk (LR) $(N=35)$. Due to the tendency of dyslexia to run strongly in families, the high-risk group was selected based upon the child having at least one first-degree family relative with an official diagnosis of dyslexia. The low-risk group was matched to the high-risk group based upon measures of intelligence, socioeconomic status, gender, age and educational environment. High-risk children were selected for participation based on their meeting the criteria: having at least one first-degree family relative possessing a formal diagnosis of a reading disability (i.e. dyslexia); possessing no signs of brain damage or longterm auditory or visual impairments; and being a native English speaker born in 2008 and entering the second year of kindergarten (SK).

Recruitment involved the distribution of letters of invitation to families of pupils a few months before entering SK. Parents were requested to complete an online questionnaire to investigate the general development, medical history and the behaviour of the participating child, along with evaluating the family history of reading and spelling (dis)abilities of the family members. The potential existence of ADHD and behavioural problems was also screened by the inclusion of a hyperactivity measure consisting of questions taken from the Strengths and Difficulties Questionnaire (SDQ; Goodman, 2001). Through information gained from the parental survey children with a history of brain damage, language problems, psychiatric symptoms, visual problems or hearing loss were excluded from the study. In addition, the questionnaire assessed the educational level through the use of the 7-point ISCED scale (International Standard Classification of Education by UNESCO, 1997). The various educational levels represented in the scale were then further simplified into three educational categories: secondary school ( $\mathrm{Sec}$ ), post-secondary (PS), graduate studies (GS). Groups were found not to differ on measures of age, IQ, hyperactivity and SES and parental educational level as seen in Table 1 .

As testing occurred within the first two and a half months of Senior Kindergarten, all participating children had not received any formal reading instruction prior to their testing date. Therefore, for the purposes of this study these children will be considered as pre-reading.

\section{Materials and procedures}

\section{Socioeconomic status}

Socioeconomic status (SES) was assessed with the Family Affluence Scale II (FAS II), developed by the World Health Organization (WHO). The FAS II is a four-part measure of family wealth scored as a composite 
Table 1 Participant characteristics

\begin{tabular}{lccc}
\hline & NR $(n=35)$ & DYS $(n=23)$ & $p$-value \\
\hline $\begin{array}{l}\text { Gender (F/M) } \\
\text { Age in months } \\
(\text { mean } \pm S D)\end{array}$ & $17 / 18$ & $12 / 11$ & $.115^{\mathrm{b}}$ \\
$\begin{array}{c}\text { Nonverbal IQ } \\
\text { (mean } \pm S D)\end{array}$ & $107.1 \pm 8.1$ & $106.0 \pm 6.3$ & $.565^{\mathrm{c}}$ \\
$\begin{array}{c}\text { Hyperactivity } \\
\text { (mean } \pm S D)\end{array}$ & $2.9 \pm 1.6$ & $3.6 \pm 2.3$ & $.205^{\mathrm{c}}$ \\
$\begin{array}{c}\text { SES (ISCED) } \\
\text { (low/middle/high) }\end{array}$ & $1 / 21 / 13$ & $2 / 13 / 8$ & $.671^{\mathrm{d}}$ \\
$\begin{array}{c}\text { Mother's education } \\
(\text { Sec/PS/GS) }\end{array}$ & $7 / 21 / 7$ & $2 / 16 / 5$ & $.588^{\mathrm{d}}$ \\
$\begin{array}{c}\text { Father's education } \\
\text { (Sec/PS/GS) }\end{array}$ & $7 / 22 / 6$ & $4 / 15 / 4$ & $1.00^{\mathrm{d}}$ \\
\hline
\end{tabular}

Notes: a Scores are standardized $(M=100, S D=15)$. ${ }^{\text {'Pearson }}$ chiSquare value. ${ }^{\text {cPaired }} t$-test. ${ }^{\mathrm{d}}$ Fisher's Exact Test. Sec $=$ secondary school education, $\mathrm{PS}=$ post-secondary education, $\mathrm{GS}=$ graduate studies.

measure ranging from 0 to 9. Similarly to Boyce, Torsheim, Currie and Zambon (2006), initial scores were transformed into three categories of low affluence (0-2), middle affluence (3-5) and high affluence (6-9).

\section{Intelligence (IQ)}

The Raven's Coloured Progressive Matrices (Raven, Raven \& Court, 1998), a collective non-verbal intelligence test measuring spatial reasoning, was administered. This test measures an individual's ability to reason and solve problems without the benefit of prior knowledge and is specifically designed for children and older adults. The test consists of 36 items in three sets, which produces a single raw score that is converted to a percentile score based on provided norms.

\section{Letter knowledge and literacy measures}

Both receptive and productive letter knowledge tests were administered through the letter writing and naming subtests of the Wide Range Achievement Test (WRAT3). For each test the 15 most frequently used letters in English books for children were used.

Productive letter knowledge. The 15 letters (printed in upper case graphemes) were presented to the child. The child was instructed to verbally produce the letter name and/or sound produced for each of the 15 graphemes. One point was attributed to each correct answer resulting in a maximum score of 15 .

Receptive letter knowledge. Fifteen common letters were presented orally. The child was required to record each spoken letter by writing it on the record sheet. A point is given for each correct response allowing for a maximum score of 15 .

Phonological awareness (PA)

A subtest of the Clinical Evaluation of Language Fundamentals 4th edn. (CELF-4) (Semel, Wiig \& Secord, 2003) was selected to assess each participant's phonological awareness ability at various grain size levels. The subtest contains 11 elements: syllable blending (SB), two syllable deletion (2SD), final syllable deletion (FSD), three syllable deletion (3SD), syllable segmentation (SS), rhyme detection (RD), rhyme production (RP), phoneme blending (PB), initial phoneme identification (IPI), medial phoneme identification (MPI), final phoneme identification (FPI).

\section{Verbal short-term memory}

The Number Repetition subtest from The Clinical Evaluation of Language Fundamentals 4th edn. (CELF-4) (Semel et al., 2003) was selected to assess verbal short-term memory. This is a digit span forward task, requiring the immediate serial recall of orally presented series of digits with lengths of 2 to 9 digits. To avoid any influence arising from differences in prior digit knowledge the subjects were asked to count from 1 to 10 to familiarize themselves with the required stimuli. All children were found to be able to complete this initial task. The test score was calculated as the total number of correct recalled lists. In addition, the non-word repetition test from the Phonological Assessment battery (PhAB; Frederickson, Frith \& Reason 1997) was employed. For this task, sequences ranging in length from two to six single syllable nonsense words were presented orally to the participants. Each participant was requested to repeat the sequence in the correct order. The sequences progressively increased in length. Students could reach a maximum score of 36 .

\section{Lexical retrieval}

Two naming tasks were administered to assess speed of phonological production through the retrieval of wholeword level phonological coding. Final scores of both tests were calculated as correctly named items per second.

A colour naming test from Boets, Wouters, van Wieringen and Ghesquière (2006) was selected for the purposes of this study. Five colours (black, yellow, red, green and blue) were presented in a random arrangement on a single sheet of paper arranged in five columns of 10 colour stimuli each. 
The object naming subtest of the Phonological Assessment Battery (PhAB) (Frederickson et al., 1997) was used. Five line drawings of common objects (desk, ball, door, hat, box) were presented in five rows, each containing 10 items.

\section{Morphological awareness}

Morphological awareness was assessed using the Wug test to evaluate the individual's ability to apply morphological change to mark inflections and derivations along with the use of appropriate possessive relationships. The 'Wug test', first developed by Berko (1958), uses target non-words which have been created to be plausible sounding English words. In the Wug test a child is shown a simple picture depicting a creature or activity and is prompted to complete a statement which requires the addition of a morpheme to the target pseudoword: 'This is a WUG. Now there is another one. There are two of them. There are two '. (Response: WUGS). A maximum score of 33 could have been obtained.

\section{Auditory processing tasks}

All tasks were conducted at the child's school and administered individually in a private room, with minimal ambient background noise and distraction. To further reduce any influence of ambient noise, over-the-ear Sennheiser HDA 200 headphones were used to present the stimuli, which offered a level of passive noise reduction. All auditory tasks were performed on a Dell Latitude D510 and controlled by APEX software (Francart, Van Wieringen \& Wouters, 2008; Laneau, Boets, Moonen, Van Wieringen \& Wouters, 2005). Auditory stimuli were presented to the right ear. Auditory processing procedure and tasks were translated from those used and described by Poelmans et al. (2011). All auditory processing task thresholds were estimated by means of a one-up, twodown adaptive staircase procedure which is designed to target a threshold corresponding to $70.7 \%$ correct responses (Levitt, 1971). Tasks were presented within a three-alternative forced-choice, 'odd-one-out', paradigm, meaning that in each trial three stimuli were presented requiring the participant to determine which sound differed from the others. An inter-stimulus interval of $350 \mathrm{~ms}$ was used. All tasks were terminated after eight reversals. The arithmetic mean of the last four reversals is used as the threshold. Each participant completed two threshold runs of each task. As the aim was to evaluate a subject's sensory capability through the use of threshold estimations, the best score of the two runs was selected (for a similar approach see Boets et al., 2006).
Frequency modulation (FM) detection. Participants were required to detect a $2 \mathrm{~Hz}$ sinusoidal frequency modulation of a $1 \mathrm{kHz}$ carrier tone with varying modulation depth. The reference stimulus was a pure tone of $1 \mathrm{kHz}$. Modulation depth decreased by a factor of 1.2 from $100 \mathrm{~Hz}$ to $11 \mathrm{~Hz}$, from which modulation depth then decreased by a step size of $1 \mathrm{~Hz}$. The length of both the reference and the target stimulus was $1000 \mathrm{~ms}$ including $50 \mathrm{~ms}$ cosine-gated onset and offset. The detection threshold was defined as the minimum depth of frequency deviation (in $\mathrm{Hz}$ ) required to detect the modulation.

Sound rise time discrimination $(R T)$. This task consisted of a speech-weighted noise with linear amplitude rise times. Rise times varied logarithmically between $15 \mathrm{~ms}$ and $699 \mathrm{~ms}$ in 50 steps. The total duration of the stimuli was fixed to $800 \mathrm{~ms}$, including a linear fall time of $75 \mathrm{~ms}$. A stimulus of $15 \mathrm{~ms}$ rise time was used as the reference stimulus for each trial. Discrimination thresholds were defined as the minimal difference in the rise time required to discriminate between the reference and target stimulus.

Intensity discrimination (ID). This task is identical to the FM and RT discrimination tasks in its presentation and procedure. Stimuli, of $800 \mathrm{~ms}$ duration, consisting of a speech-weighted noise and a linear rise time and fall time of $75 \mathrm{~ms}$ were used. The stimulus of $70 \mathrm{~dB}$ SPL was the reference stimulus for each trial. Intensity varied linearly between $70 \mathrm{~dB}$ SPL and $80 \mathrm{~dB}$ SPL in 40 steps of $0.25 \mathrm{~dB}$ SPL each. Discrimination thresholds were defined as the minimal intensity difference (in dB SPL) required to discriminate between the reference and the target stimulus. Being a non-temporal task, the ID task was used as a control variable to correct for psychophysical task demands.

\section{Statistical analyses}

Statistical analyses were performed with SPSS 20.0 software (IBM Corp., 2011). Data of all variables were checked with Shapiro-Wilk's test for normality. The assumption of homogeneity of variance was assessed by Levene's Test for Equality of Variances. Differences between HR and LR groups were investigated based on a paired $t$-test. Bonferroni adjusted alpha levels for each planned comparison were utilized in an effort to avoid the likelihood of false positive conclusions. Bonferroni adjusted alpha levels were determined by dividing the standard alpha of 0.05 by the total number of comparisons per question. The null hypothesis was rejected 
in situations where the $p$-value was less than the adjusted alpha.

Partial Pearson correlations were calculated in order to determine the relation between auditory tasks and PA as well as the relation between measures of MA and PA. Separate Bonferroni adjusted alpha levels for the auditory and PA comparisons and the planned MA and PA comparisons were constructed and reported below. Finally, group differences on MA and PA tasks were re-examined including $\mathrm{PA}$ and MA, respectively, as control variables.

\section{Results}

\section{Letter knowledge}

Results of both the productive and receptive letter knowledge tasks are found in Table 2. Significant group differences, based on an Bonferroni adjusted alpha of 0.025 , were not found for either measure, $t(23.489)=$ $-1.807 ; p=.084$, and $t(56)=-1.555 ; p=.125$. However, further examination of these results showed the

Table 2 Literacy and cognitive variables: descriptive statistics and independent t-tests results

\begin{tabular}{|c|c|c|c|c|c|c|}
\hline \multirow[b]{2}{*}{ Measure } & \multicolumn{2}{|l|}{ NR } & \multicolumn{2}{|l|}{ DYS } & \multirow[b]{2}{*}{$t$} & \multirow[b]{2}{*}{$p$} \\
\hline & $M$ & $S D$ & $M$ & $S D$ & & \\
\hline \multicolumn{7}{|l|}{ Letter knowledge } \\
\hline $\begin{array}{l}\text { Receptive } \\
\text { (WRAT-III) }\end{array}$ & 14.8 & 0.5 & 14 & 2.1 & -1.807 & .084 \\
\hline $\begin{array}{l}\text { Productive } \\
\text { (WRAT-III) }\end{array}$ & 13.5 & 2.2 & 12.3 & 3.4 & -1.555 & .125 \\
\hline $\begin{array}{l}\text { Morphological } \\
\text { awareness }\end{array}$ & 21.7 & 3.8 & 18.4 & 4.4 & -3.017 & $.004 *$ \\
\hline \multicolumn{7}{|c|}{ Phonological measures } \\
\hline RANcomp & 0.0 & 0.9 & -0.5 & 0.8 & -2.268 & .027 \\
\hline VSTMcomp & 0.0 & 0.9 & -0.5 & 0.8 & -2.292 & .026 \\
\hline PAcomp & 0.3 & 0.6 & -0.5 & 0.7 & -4.925 & $<.001 *$ \\
\hline $\begin{array}{l}\text { Syllable } \\
\text { awareness }\end{array}$ & 18.4 & 2.6 & 15.4 & 2.7 & -4.143 & $<.001 *$ \\
\hline $\begin{array}{l}\text { Rhyme } \\
\text { awareness }\end{array}$ & 8.6 & 1.7 & 6.9 & 2.3 & 3.359 & $.001 *$ \\
\hline $\begin{array}{l}\text { Phoneme } \\
\text { awareness }\end{array}$ & 14.4 & 4.0 & 10.6 & 5.4 & 2.630 & $.011^{*}$ \\
\hline \multicolumn{7}{|c|}{ Auditory temporal processing } \\
\hline Rise Time $(\mathrm{ms})$ & 299.7 & 184.3 & 408.0 & 178.6 & 2.216 & .031 \\
\hline $\begin{array}{l}\text { Frequency } \\
\text { modulation } \\
(\mathrm{Hz})\end{array}$ & 10.9 & 4.6 & 11.1 & 5.9 & .191 & .849 \\
\hline $\begin{array}{l}\text { Intensity } \\
\text { discrimination } \\
\text { (dB) }\end{array}$ & 3.3 & 1.3 & 3.8 & 1.8 & 1.035 & .307 \\
\hline
\end{tabular}

Notes: * $p$-values were found to be less than Bonferroni adjusted alpha levels. potential influence of a lack of variance within each measure due to ceiling effects. Exploration of the results revealed that $91 \%$ and $74 \%$ of participants produced one or fewer errors on the receptive and productive letter knowledge tasks.

Phonological skills in high and low family risk children

To answer the question whether the high-risk group demonstrates a deficit in the phonological domain, group differences of three aspects of phonological processing are tested. Component scores were created for each sub-skill: PA, verbal short-term memory, and lexical retrieval (RAN). $Z$-scores for all RAN and verbal short-term memory tasks were created based on control group mean and standard deviation. The RAN component score (RANcomp) was the average of both object and colour naming $\mathrm{z}$-scores. The verbal short-term memory component score (VSTMcomp) was the average of the number repetition and non-word repetition subtest $z$-scores. The phonological awareness component score (PAcomp) was created by averaging total scores for syllable (SB, 2SD, FSD, 3SD, SS), rhyme (RD, RP), and phoneme awareness sub-test scores (PB, IPI, MPI, FPI).

Independent sample $t$-tests were performed to determine differences between groups in measures of phonological skills and used Bonferroni adjusted alpha levels of $0.017(0.05 / 3)$. All scores were found to be normally distributed. High-risk children were found to perform significantly poorer than low-risk controls on the component score of PA. Yet RAN and verbal short-term memory were not found to be significant when Bonferroni adjusted alpha of 0.017 was utilized. All results of group comparisons, along with group mean and standard deviation scores, for each component score can be found in Table 2.

To further understand performance differences on specific phonological sub-skills, group differences across the various grain sizes of PA were examined. In order to approach a normal distribution the negatively skewed variables, rhyme and phoneme awareness, were logarithmically transformed after the scores had been reversed (Field, 2009). Results demonstrated that high-risk readers scored significantly poorer at all levels of phonological awareness (adjusted $\alpha=0.017$ ). Results of the group comparisons can be found in Table 2 .

\section{Auditory temporal processing and its relationship with $P A$}

To evaluate the proposed auditory temporal processing deficit theory of dyslexia in a sample of high- and low- 
risk pre-reading children, the various grain sizes of PA in relation to measures of RT and FM were studied. Firstly, utilizing an unadjusted standard alpha of 0.05 , group comparisons demonstrated that high-risk children scored significantly poorer than controls on measures of RT discrimination. Group differences were not found for FM detection nor for the control ID task. Yet with the application of Bonferroni adjusted alpha of 0.017 , group differences for RT did not remain significant.

To assess the relationships between subjects' phonological awareness and auditory processing skills, Pearson's correlation coefficients were calculated between the subjects' scores on measures of syllable awareness, rhyme awareness, phoneme awareness, and measures of slowrate dynamic auditory processing. Partial correlations were conducted to control for effect of group, and Bonferroni adjusted alpha levels of $0.008(0.05 / 6)$ and $0.0017(0.01 / 6)$ were used to account for multiple comparisons. No significant correlations between measures of phonological awareness and auditory temporal processing were found. Yet a relation between syllable awareness and the measure of RT was found to be approaching significance $(p<.05)$ (see Table 3 ).

\section{The relationship between pre-reading morphological awareness and $P A$}

As reported in Table 2, a significant group difference was found for the measure of morphological awareness $(\alpha=0.05)$. As poor MA of older dyslexic children is often attributed to their poor PA skills, relationships of MA and the various grain sizes of PA were examined. As reported in Table 3, Pearson correlations were performed to examine these relationships with Bonferroni adjusted alpha levels of $0.017(0.05 / 3)$ and $0.003(0.01 / 3)$ to account for multiple comparisons. MA was found to be significantly related to syllable awareness $(p<.003)$.

Due to the theoretical influence of PA on the attainment of morphological awareness and the observed significant correlation of MA and syllable awareness, a

Table 3 Partial correlations between measures of PA, MA and auditory processing, controlled for group membership

\begin{tabular}{lrrr}
\hline & \multicolumn{4}{l}{ Auditory processing } \\
\cline { 2 - 4 } & RT & FM & MA \\
\hline Syllable awareness & $-.306^{\wedge}$ & -.161 & $.341^{\text {oo }}$ \\
Rhyme awareness & .058 & .000 & .131 \\
Phonemic awareness & -.020 & -.056 & .143 \\
\hline
\end{tabular}

Note: Level of significance was obtained after Bonferroni adjustment. Auditory processing and $\mathrm{PA}:{ }^{\wedge} p<.05$.

Morphological awareness (MA) and PA: ${ }^{\circ} p<.017 ;{ }^{\circ o} p<.003$. series of ANCOVAs were run to determine the effect of group differences while controlling for these variables. After introducing PAcomp as a covariate, no significant difference could be found of the measure of morphological awareness between groups, $F(1,55)=3.171, p=.080$, partial $\eta^{2}=.055$. Similarly, the inclusion of syllable awareness as a covariate removed any group difference on the MA task, $F(1,55)=3.632, p=.062$, partial $\eta^{2}=.062$.

To further understand the relationship of MA and PA at the pre-reading stage of development, the reverse of the above ANCOVAs were preformed, with MA as the covariate. Results showed that group differences remained for both PAcomp and syllable awareness scores while controlling for MA, $F(1,55)=13.518, p=.001$, partial $\eta^{2}=.197$ and $F(1,55)=7.961, p=.007$, partial $\eta^{2}=.126$.

\section{Discussion}

In a group of pre-reading children with a family risk of dyslexia, this study investigated how the phonological deficit is expressed at various grain sizes of PA and whether these grain sizes are related to measures of auditory temporal processing. Moreover, the study attempted to shed light on the pre-reading relationship of MA and PA. Studying this relationship in a prereading population allows us to disentangle the relationship of PA and MA from reading experience.

\section{Phonological processing deficit}

As we don't yet know which children will be classified as dyslexic later on, the design of the study relies on comparisons of children with a family risk for developing dyslexia. In addition, it was not possible to fully disentangle environmental and instructional factors, yet the groups were balanced for age, gender, IQ, socioeconomic status, parental education, and letter knowledge. Although we have evaluated an at-risk sample, results were in line with what would have been expected for a dyslexic sample. The three aspects of phonological processing, phonological awareness, verbal short-term memory, and lexical retrieval, were able to differentiate between pre-reading children with a family risk of dyslexia and low-risk children. These results are also consistent with similar research that revealed deficits in pre-reading children with a family risk of dyslexia (Elbro et al., 1998; Gallagher, Frith \& Snowling, 2000; Pennington \& Lefly, 2001; Snowling et al., 2003). Yet, it is worth noting that the group differences on the factors RANcomp and VSTMcomp could not withstand 
Bonferroni correction for multiple testing. This was in line with the findings of the prospective studies of Boets et al. (2006) and Elbro et al. (1998), while others, though using a retrospective analysis of their population, could find significant differences on measures of verbal shortterm memory and RAN (de Jong \& van der Leij, 2003; Pennington \& Lefly, 2001).

\section{$P A$ at various grain sizes}

Phonological awareness is believed to progressively develop from larger grain size units of syllables through rhyme awareness to the smallest grain size of phoneme awareness. The progression to an explicit awareness of this smallest unit is thought to only manifest after explicit instruction (Ziegler \& Goswami, 2005). As a result of this developmental sequence, we hypothesized that group differences would be prominent for the larger grain units of syllable and rhyme, while phonemic awareness was predicted to be still underdeveloped in both groups.

As predicted, significant group differences were found for both syllable and rhyme awareness, yet unexpectedly group differences were also found for phoneme awareness. Since phoneme awareness has been said to only manifest as a result of explicit instruction, the discovery of developed phoneme awareness is a reasonable finding in light of the large proportion of the sample performing at ceiling level on the letter knowledge tasks. Such results suggest some level of formal or informal literacy instruction, or at least knowledge of the alphabet.

Since phonological awareness has been consistently demonstrated to be one of the best pre-school predictors of reading achievement, it is reasonable to assume from our findings that the family risk group will contain a larger proportion of future cases of dyslexia compared to the LR group.

\section{Auditory temporal processing and its relation with $P A$}

We examined RT discrimination and FM detection in order to investigate slow-rate auditory temporal processing. We hypothesized that our findings would be in line with the slow-rate auditory temporal processing theory, in that both FM and RT would differentiate between both groups, but not our non-temporal auditory ID task. However, a significant difference in auditory temporal processing between the HR and the LR group was only observed for the measure of RT discrimination and not for FM. As expected, no group difference was found for intensity discrimination, the task that was used to control for task demands during assessment.
Although group differences for FM measures have been observed in an adult population (Heath et al., 2006; Ramus et al., 2003; Witton et al., 1998; but see Law et al. 2014), our findings in a pre-reading sample supported the findings of Boets et al. (2006). Boets and colleagues proposed that the lack of significant group differences for the FM measures may be attributed to either the typically greater inter-individual variability in children or to a poorly defined clinical group which may have contained substantial overlap with the control group. Although both are plausible arguments, the contrasting findings of a significant group difference of RT and the lack of difference of FM in the same sample require further explanation. We suggest two plausible hypotheses explaining these contrasting findings: (1) differences may have resulted from sensitivity differences between measures, yet more likely (2) differences in the specific characteristics of the auditory stimuli being used could be to blame. As discussed by Law et al. (2014), it is most likely that the inconsistency between RT and FM results can be linked to the fact that both tasks represent different aspects of the auditory information in the speech signal. While FM represents the fine structure of the speech waveform, RT represents amplitude aspects of the speech envelope. Supporting the conclusion of the adult study by Law et al. (2014), our pre-reading results suggest that the primary auditory dysfunction in dyslexics is not to be found in the processing of the fine structure of the speech wave form but more likely in the perception of slow-rate dynamic auditory cues related to the speech envelope, as measured by the RT task. Such findings reinforce previous studies in both child and adult populations (Fraser, Goswami \& Conti-Ramsden, 2010; Goswami et al., 2002; Law et al., 2014; Poelmans et al., 2011; Thomson et al., 2006).

In summary, our findings do not support a general deficit in slow-rate auditory processing of dyslexics; nevertheless, dyslexics may have a more specific slow-rate dynamic auditory processing deficit specific to the envelopes of the speech waveform.

\section{Relation between auditory processing measures and PA}

An analysis of the relation between auditory processing measures and the various grain sizes of PA was carried out. FM showed no significant correlations with any of the three PA measures; a trend towards a RT and syllable awareness relationship was observed. Theoretically, the relationship between RT and syllable awareness is sensible, due to the characteristics of the RT task. As discussed earlier, the stimuli used in the RT tasks are designed to correspond to time windows related to 
speech envelope cues that are supra-segmental. These cues are thought to facilitate syllable segmentation of the acoustic-linguistic signal. Thus it is more reasonable to find correlations between a measure of RT and syllable awareness than a smaller grain size unit of PA.

Although our research design does not permit any conclusions relating to the directionality of this relationship, a plausible prediction can be made. Hämäläinen et al. (2005) discussed that it is highly improbable that an individual's poor phonological awareness would be influential on the lower-level skills of rise time discrimination. Hence, this relationship either reflects the same underlying perceptual deficit, or the auditory processing ability underlining the RT task has a causal role in the development of PA.

\section{The relationship between pre-reading morphological awareness and $P A$}

We explored the pre-reading relation of MA and the various measures of PA. The deficit of MA observed in dyslexics is often treated as consequential to the dyslexic reader's poor reading experience that in itself is a consequence of their poor PA skills. In order to disentangle this relationship, we evaluated these measures in a pre-reading population to remove the influence of reading experience.

It was hypothesized that MA would be related to PA prior to reading instruction. As a consequence we predicted that any observed deficit in PA in pre-readers would produce an observable group difference in MA between high- and low-risk children.

Correlational analysis demonstrated a relation between MA and the composite score of PA and syllable awareness. Group comparisons confirmed our hypothesis, demonstrating that prior to any reading experience children at risk of dyslexia have a relative deficit in MA. To help further our understanding of this relationship, group differences of MA and PA were re-examined including PA and MA respectively as a control variable. After controlling for PA, group differences for the MA task were no longer present while group differences in PA were preserved when MA was added as a co-variant.

Our results support the research findings by Cunningham and Carroll (2013) and Carlisle and Nomanbhoy (1993) who reported a relation between pre-reading PA and later MA in that pre-reading PA predicted later MA. Our study differed in the sense that MA was assessed prior to the onset of formal reading instruction which allowed for disentangling reading experience from this relationship. As a result, we can conclude that PA is a relevant component of MA, independent of reading experience. Therefore the poor PA of dyslexic children, independent of reading experience, may be attributed to the observed MA deficit in dyslexic readers.

This study has demonstrated that children with a family risk of dyslexia possess reduced MA skills when compared to controls. As MA has been shown to be an important variable in predicting later word reading and reading comprehension, then a disruption or delay in MA development could potentially exacerbate later reading problems of children with dyslexia.

Although studies on adults and the results of this study have demonstrated MA deficits in individuals with dyslexia, evidence from intervention studies have demonstrated that direct instruction of MA does have a beneficial influence on bridging the MA achievement gap of dyslexic children (for a review see Bowers et al., 2010). This suggests that although early MA development is influenced by the child's phonological deficit, direct instruction and the development of a more explicit knowledge of the morphological structure of language has the potential to overcome this influence. In addition, several studies have suggested MA as a potential compensatory variable to overcome the phonological deficit and aid in reading achievement (Elbro \& Arnbak, 1996; Law et al., 2015; Leikin \& Zur Hagit, 2006). For MA to be considered as a means of compensation, Law et al. (2015) noted two conditions which must be satisfied: MA must be found to be independent of PA, as well as MA being intact and a strength for individuals with dyslexia. Yet according to the findings of this paper neither of these conditions is capable of being met within a pre-reading population. If these conditions were to be met to support the findings of Law et al. (2015) the observed pre-reading influence of PA would need to diminish throughout development. A possible scenario could be that through literacy instruction a more explicit form of MA develops coupled with the development of orthographic representations of the morphemes. Such explicit awareness and representations could result in a new developmental trajectory independent of PA. Further longitudinal research spanning the pre- and post-reading instructional phases of development is required to fully understand the influence that direct literacy instruction has on the developmental trajectory of MA.

\section{References}

Berko, J. (1958). The child's learning of English morphology. Doctoral dissertation, Radcliffe College.

Boets, B., Vandermosten, M., Poelmans, H., Luts, H., Wouters, J. et al. (2011). Preschool impairments in auditory processing and speech perception uniquely predict future reading 
problems. Research in Developmental Disabilities, 32 (2), 560570.

Boets, B., Wouters, J., van Wieringen, A., \& Ghesquière, P. (2006). Auditory temporal information processing in preschool children at family risk for dyslexia: relations with phonological abilities and developing literacy skills. Brain and Language, 97 (1), 64-79. doi:10.1016/ j.band1.2005.07.026

Boets, B., Wouters, J., Van Wieringen, A., \& Ghesquière, P. (2007). Auditory processing, speech perception and phonological ability in pre-school children at high-risk for dyslexia: a longitudinal study of the auditory temporal processing theory. Neuropsychologia, 45 (8), 1608-1620.

Bowers, P.N., Kirby, J.R., \& Deacon, S.H. (2010). The effects of morphological instruction on literacy skills: a systematic review of the literature. Review of Educational Research, 80 (2), 144-179.

Boyce, W., Torsheim, T., Currie, C., \& Zambon, A. (2006). The family affluence scale as a measure of national wealth: validation of an adolescent self-report measure. Social Indicators Research, 78 (3), 473-487.

Bryant, P.E., MacLean, M., Bradley, L.L., \& Crossland, J. (1990). Rhyme and alliteration, phoneme detection, and learning to read. Developmental Psychology, 26 (3), 429-438.

Caravolas, M., Hulme, C., \& Snowling, M.J. (2001). The foundations of spelling ability: evidence from a 3-year longitudinal study. Journal of Memory and Language, 45 (4), 751-774.

Carlisle, J.F. (1995). Morphological awareness and early reading achievement. In L.B. Feldman (Ed.), Morphological Aspects of Language Processing (pp. 189-209). Hillsdale, NJ: Erlbaum.

Carlisle, J.F. (2000). Awareness of the structure and meaning of morphologically complex words: impact on reading. Reading and Writing, 12 (3), 169-190.

Carlisle, J.F. (2004). Morphological processes that influence learning to read. In C.A. Stone, E.R. Silliman, B.J. Ehren, \& K. Apel (Eds.), Handbook of language and literacy (pp. 318339). New York: Guilford Press.

Carlisle, J.F., \& Fleming, J. (2003). Lexical processing of morphologically complex words in the elementary years. Scientific Studies of Reading, 7 (3), 239-253.

Carlisle, J.F., \& Nomanbhoy, D.M. (1993). Phonological and morphological awareness in first graders. Applied Psycholinguistics, 14 (02), 177-195.

Casalis, S., \& Colé, P. (2009). On the relationship between morphological and phonological awareness: effects of training in kindergarten and in first-grade reading. First Language, 29 (1), 113-142.

Casalis, S., Colé, P., \& Sopo, D. (2004). Morphological awareness in developmental dyslexia. Annals of Dyslexia, 54 (1), 114-138.

Chiat, S. (2001). Mapping theories of developmental language impairment: premises, predictions and evidence. Language and Cognitive Processes, 16 (2-3), 113-142.

Corriveau, K.H., Goswami, U., \& Thomson, J.M. (2010). Auditory processing and early literacy skills in a preschool and kindergarten population. Journal of Learning Disabilities, 43 (4), 369-382.

Corriveau, K., Pasquini, E., \& Goswami, U. (2007). Basic auditory processing skills and specific language impairment: a new look at an old hypothesis. Journal of Speech, Language, and Hearing Research, 50 (3), 647-666.

Cunningham, A.J., \& Carroll, J.M. (2013). Early predictors of phonological and morphological awareness and the link with reading: evidence from children with different patterns of early deficit. Applied Psycholinguistics, 36 (03), 509-531.

Dandache, S., Wouters, J., \& Ghesquière, P. (2014). Development of reading and phonological skills of children at family risk for dyslexia: a longitudinal analysis from kindergarten to sixth grade. Dyslexia, 20 (4), 305-329.

Dawes, P., \& Bishop, D. (2009). Auditory processing disorder in relation to developmental disorders of language, communication and attention: a review and critique. International Journal of Language Communication Disorders, 44 (4), 440 465. doi:10.1080/13682820902929073

Deacon, S.H., \& Kirby, J.R. (2004). Morphological awareness: Just 'more phonological'? The roles of morphological and phonological awareness in reading development. Applied Psycholinguistics, 25 (02), 223-238.

Deacon, S.H., Parrila, R., \& Kirby, J.R. (2008). A review of the evidence on morphological processing in dyslexics and poor readers: a strength or weakness? In V. Berninger, A. Fawcett, G. Reid, \& L. Siegel (Eds.), The Sage handbook of dyslexia (pp. 212-237). Thousand Oaks, CA: Sage.

de Jong, P.F., \& van der Leij, A. (2003). Developmental changes in the manifestation of a phonological deficit in dyslexic children learning to read a regular orthography. Journal of Educational Psychology, 95 (1), 22-40.

Elbro, C. (1989). Morphological awareness in dyslexia. In C. von Euler, I. Lundberg, \& G. Lennerstrand (Eds.), Brain and reading (pp. 279-291). New York: Springer.

Elbro, C., \& Arnbak, E. (1996). The role of morpheme recognition and morphological awareness in dyslexia. Annals of Dyslexia, 46 (1), 209-240. doi:10.1007/BF02648177

Elbro, C., Borstrøm, I., \& Petersen, D.K. (1998). Predicting dyslexia from kindergarten: the importance of distinctness of phonological representations of lexical items. Reading Research Quarterly, 33, 36-60. doi:10.1598/RRQ.33.1.3

Field, A.P. (2009). Discovering statistics Using SPSS (and sex and drugs and rock ' $n$ ' roll) (3rd edn.). London: Sage.

Fowler, A.E., \& Liberman, I.Y. (1995). The role of phonology and orthography in morphological awareness. In L.B. Feldman (Ed.), Morphological aspects of language processing (pp. 157-188). Hillsdale, NJ: Erlbaum.

Francart, T., Van Wieringen, A., \& Wouters, J. (2008). APEX 3: a multi-purpose test platform for auditory psychophysical experiments. Journal of Neuroscience Methods, 172 (2), 283 293.

Fraser, J., Goswami, U., \& Conti-Ramsden, G. (2010). Dyslexia and specific language impairment: the role of phonology and auditory processing. Scientific Studies of Reading, 14 (1), 829. 
Frederickson, N., Frith, U., \& Reason, R. (1997). Phonological Assessment Battery (Manual and test materials). Windsor: NFER-Nelson.

Gallagher, A., Frith, U., \& Snowling, M.J. (2000). Precursors of literacy delay among children at genetic risk of dyslexia. Journal of Child Psychology and Psychiatry, 41 (2), 203-213.

Goodman, R. (2001). Psychometric properties of the Strengths and Difficulties Questionnaire. Journal of the American Academy of Child \& Adolescent Psychiatry, 40 (11), 13371345.

Goswami, U., Fosker, T., Huss, M., Mead, N., \& Szucs, D. (2011). Rise time and formant transition duration in the discrimination of speech sounds: the $\mathrm{Ba}-\mathrm{Wa}$ distinction in developmental dyslexia. Developmental Science, 14 (1), 34 43. doi:10.1111/j.1467-7687.2010.00955.x

Goswami, U., Thomson, J., Richardson, U., Stainthorp, R., Hughes, D. et al. (2002). Amplitude envelope onsets and developmental dyslexia: a new hypothesis. Proceedings of the National Academy of Sciences, USA, 99 (16), 10911-10916. doi:10.1073/pnas.122368599

Hämäläinen, J., Leppänen, P.H.T., Torppa, M., Müller, K., \& Lyytinen, H. (2005). Detection of sound rise time by adults with dyslexia. Brain and Language, 94 (1), 32-42.

Hämäläinen, J.A., Salminen, H.K., \& Leppänen, P.H. (2013). Basic auditory processing deficits in dyslexia: systematic review of the behavioral and event-related potential/field evidence. Journal of Learning Disabilities, 46 (5), 413-427.

Heath, S.M., Bishop, D.V.M., Hogben, J.H., \& Roach, N.W. (2006). Psychophysical indices of perceptual functioning in dyslexia: a psychometric analysis. Cognitive Neuropsychology, 23, 905-929.

Kieffer, M.J., \& Lesaux, N.K. (2012). Direct and indirect roles of morphological awareness in the English reading comprehension of native English, Spanish, Filipino, and Vietnamese speakers. Language Learning, 62 (4), 1170-1204.

Kirby, J.R., Deacon, S.H., Bowers, P.N., Izenberg, L., WadeWoolley, L. et al. (2012). Children's morphological awareness and reading ability. Reading and Writing, 25 (2), 389-410.

Kirk, C., \& Gillon, G.T. (2007). Longitudinal effects of phonological awareness intervention on morphological awareness in children with speech impairment. Language, Speech, and Hearing Services in Schools, 38 (4), 342-352.

Laneau, J., Boets, B., Moonen, M., Van Wieringen, A., \& Wouters, J. (2005). A flexible auditory research platform using acoustic or electric stimuli for adults and young children. Journal of Neuroscience Methods, 142 (1), 131-136.

Law, J.M., Vandermosten, M., Ghesquière, P., \& Wouters, J. (2014). The relationship of phonological ability, speech perception, and auditory perception in adults with dyslexia. Frontiers in Human Neuroscience, 8, 482.

Law, J.M., Wouters, J., \& Ghesquière, P. (2015). Morphological awareness and its role in compensation in adults with dyslexia. Dyslexia, 21 (3), 254-272. doi:10.1002/dys.1495

Leikin, M., \& Zur Hagit, E. (2006). Morphological processing in adult dyslexia. Journal of Psycholinguistic Research, 35 (6), 471-490.
Levitt, H. (1971). Transformed up-down methods in psychoacoustics. Journal of the Acoustical Society of America, 49 (2B), 467-477.

Lyytinen, P., \& Lyytinen, H. (2004). Growth and predictive relations of vocabulary and inflectional morphology in children with and without familial risk for dyslexia. Applied Psycholinguistics, 25 (03), 397-411.

McBride-Chang, C., Wagner, R.K., Muse, A., Chow, B.W.-Y., \& Shu, H. (2005). The role of morphological awareness in children's English reading and vocabulary acquisition. Applied Psycholinguistics, 26, 415-435.

Nagy, W.E., \& Anderson, R.C. (1984). How many words are there in printed school English? Reading Research Quarterly, 19 (3), 304-330.

Nagy, W., Berninger, V.W., \& Abbott, R.D. (2006). Contributions of morphology beyond phonology to literacy outcomes of upper elementary and middle-school students. Journal of Educational Psychology, 98 (1), 134-147.

Obrig, H., Rossi, S., Telkemeyer, S., \& Wartenburger, I. (2010). From acoustic segmentation to language processing: evidence from optical imaging. Frontiers in Neuroenergetics, 2 , 13.

Pennington, B.F. (2006). From single to multiple deficit models of developmental disorders. Cognition, 101 (2), 385-413.

Pennington, B.F., \& Lefly, D.L. (2001). Early reading development in children at family risk for dyslexia. Child Development, 72 (3), 816-833.

Poelmans, H., Luts, H., Vandermosten, M., Boets, B., Ghesquière, P. et al. (2011). Reduced sensitivity to slow-rate dynamic auditory information in children with dyslexia. Research in Developmental Disabilities, 32 (6), 2810-2819.

Ramus, F. (2001). Outstanding questions about phonological processing in dyslexia. Dyslexia, 7, 197-216. doi:10.1002/ dys. 205

Ramus, F., Rosen, S., Dakin, S.C., Day, B.L., Castellote, J.M. et al. (2003). Theories of developmental dyslexia: insights from a multiple case study of dyslexic adults. Brain, 126 (4), $841-865$.

Ramus, F., \& Szenkovits, G. (2008). What phonological deficit? Quarterly Journal of Experimental Psychology, 61 (1), 129141.

Raven, J., Raven, J.C., \& Court, J.H. (1998). Raven's progressive matrices and vocabulary scales. Oxford: Oxford Psychologists Press.

Roman, A.A., Kirby, J.R., Parrila, R.K., Wade-Woolley, L., \& Deacon, S.H. (2009). Toward a comprehensive view of the skills involved in word reading in grades 4, 6, and 8. Journal of Experimental Child Psychology, 102 (1), 96-113.

Semel, E., Wiig, E.H., \& Secord, W.A. (2003). Clinical Evaluation of Language Fundamentals (CELF-4th edn.). San Antonio, TX: Harcourt Assessment.

Shankweiler, D., Crain, S., Katz, L., Fowler, A., Liberman, A. et al. (1995). Cognitive profiles of reading-disabled children: comparison of language skills in phonology, morphology, and syntax. Psychological Science, 6 (3), 149-156.

Singson, M., Mahony, D., \& Mann, V. (2000). The relation between reading ability and morphological skills: evidence 
from derivational suffixes. Reading and Writing, 12 (3), 219252.

Snowling, M.J. (2000). Dyslexia. Oxford: Wiley-Blackwell.

Snowling, M.J., Gallagher, A., \& Frith, U. (2003). Family risk of dyslexia is continuous: individual differences in the precursors of reading skill. Child Development, 74 (2), 358373.

Snowling, M.J., Muter, V., \& Carroll, J. (2007). Children at family risk of dyslexia: a follow-up in early adolescence. Journal of Child Psychology and Psychiatry, 48 (6), 609-618.

SPSS (2011). IBM SPSS statistics base 20. Chicago, IL: SPSS Inc.

Tallal, P. (1980). Auditory temporal perception, phonics and reading disabilities in children. Brain and Language, 9, 182198.

Thomson, J.M., Fryer, B., Maltby, J., \& Goswami, U. (2006). Auditory and motor rhythm awareness in adults with dyslexia. Journal of Research in Reading, 29 (3), 334-348.

Tønnessen, F.E. (1997). How can we best define 'dyslexia'? Dyslexia, 3 (2), 78-92.

UNESCO (1997). International Standard Classification of Education-ISCED 1997: November 1997. Paris: UNESCO.

Van Ingelghem, M., Boets, B., van Wieringen, A., Onghena, P., Ghesquière, P. et al. (2005). An auditory temporal processing deficit in children with dyslexia? In P. Ghesquière \& A.J.J.M. Ruijssenaars (Eds.), Children with learning disabilities: $A$ challenge to teaching and instruction (pp. 47-63). Leuven, Belgium: Leuven University Press.

Vanvooren, S., Poelmans, H., Hofmann, M., Ghesquière, P., \& Wouters, J. (2014). Hemispheric asymmetry in auditory processing of speech envelope modulations in prereading children. Journal of Neuroscience, 34 (4), 1523-1529.
Vellutino, F.R., Fletcher, J.M., Snowling, M.J., \& Scanlon, D.M. (2004). Specific reading disability (dyslexia): what have we learned in the past four decades? Journal of Child Psycholology and Psychiatry, 45, 2-40.

Wagner, R.K., \& Torgesen, J.K. (1987). The nature of phonological processing and its causal role in the acquisition of reading skills. Psychological Bulletin, 101 (2), 192-212.

Wagner, R.K., Torgesen, J.K., \& Rashotte, C.A. (1994). Development of reading-related phonological processing abilities: new evidence of bidirectional causality from a latent variable longitudinal study. Developmental Psychology, 30 (1), 73-87.

Witton, C., Stein, J.F., Stoodley, C.J., Rosner, B.S., \& Talcott, J.B. (2002). Separate influences of acoustic AM and FM sensitivity on the phonological decoding skills of impaired and normal readers. Journal of Cognitive Neuroscience, 14 (6), 866-874.

Witton, C., Talcott, J.B., Hansen, P.C., Richardson, A.J., Griffiths, T.D. et al. (1998). Sensitivity to dynamic auditory and visual stimuli predicts nonword reading ability in both dyslexic and normal readers. Current Biology, 8, 791-797.

Woodcock, R.W., McGrew, K.S., \& Mather, N. (2001). Woodcock-Johnson tests of achievement. Itasca, IL: Riverside Publishing.

Ziegler, J.C., \& Goswami, U. (2005). Reading acquisition, developmental dyslexia, and skilled reading across languages: a psycholinguistic grain size theory. Psychological Bulletin, 131 (1), 3-29.

Received: 11 May 2015

Accepted: 15 April 2016 